\title{
The Diagonalization Paradox
}

\section{Cantor's Diagonal Method Can Lead to Conflicting Results \\ Ron Ragusa}

May 2020

\begin{abstract}
In 1891 Georg Cantor published his Diagonal Method which, he asserted, proved that the real numbers cannot be put into a one-to-one correspondence with the natural numbers. In this paper we will see how by varying the initial conditions of Cantor's proof we can use the diagonal method to produce a one-to-one correspondence between the set of natural numbers and the set of infinite binary decimals in the interval $(0,1)$.

In the appendix we demonstrate that using the diagonal method recursively will, at the limit of the process, fully account for all the infinite binary decimals in $(0,1)$. The proof will cement the one-to-one correspondence between the natural numbers and the infinite binary decimals in $(0,1)$.
\end{abstract}




\section{Introduction}

In 1891 Georg Cantor published his Diagonal Method which, he asserted, proved that the real numbers cannot be put into a one-to-one correspondence with the natural numbers. Cantor's proof relies on the fact that a complete list of infinite binary decimals over the open interval $(0,1)$ cannot, in fact be realized. That is to say, the infinite set of binary decimal numbers in $(0,1)$ cannot be listed linearly in its entirety and thus is uncountable. Cantor's reasoning is that since the binary decimal numbers listed, even though infinite, must necessarily be incomplete, then any attempt to put those numbers in $(0,1)$ in a one-to-one correspondence with the natural numbers is bound to fail. To demonstrate, we begin with a matched list of infinite binary decimal numbers in $(0,1)$ which we assume is complete. Each number in the list is matched with a corresponding natural number. Any infinite binary decimal number not in the list will have no natural number to pair with. Cantor's diagonal method produces numbers not contained in the list and from this he concludes that the set of infinite binary decimals in $(0,1)$ must have more members than the set of all natural numbers.

In this paper we will see how by varying the initial conditions of Cantor's proof we can use the diagonal method to produce a one-to-one correspondence between the set of natural numbers and the set of infinite binary decimals in $(0,1)$. We concede that the initial list of infinite binary decimals is, in fact, incomplete and that the diagonal method does produce a number not contained in the list. Also we'll agree that there are an infinite number of binary decimal numbers in the interval that are not in the list. We are not arguing that Cantor's proof is incorrect, only that it's not the last word on the subject. We will see how using the same diagonal method we can create infinitely many binary decimal numbers contained in the interval and that for each number we so create, each will correspond with one and only one natural number.

In the appendix we demonstrate that using the diagonal method recursively will, at the limit of the process, fully account for all the infinite binary decimals in $(0,1)$. The proof will cement the one-to-one correspondence between the natural numbers and the infinite binary decimals in $(0,1)$. 


\section{Initial Conditions, Cantor's 1891 Proof}

1. The set $B$ of infinite binary decimals on the interval $(0,1)$,

$$
B=\{d: 0<d<1\}
$$

2. An assumed complete list $L$, of the elements of $B$.

\begin{tabular}{llllllllllll}
\multicolumn{11}{c}{$\mathrm{d}$} \\
\hline. & 0 & 1 & 0 & 1 & 0 & 1 & 0 & 1 & 0 & 1 & $\ldots$ \\
. & 1 & 1 & 0 & 1 & 1 & 0 & 1 & 1 & 0 & 1 & $\ldots$ \\
. & 0 & 0 & 1 & 0 & 0 & 1 & 0 & 0 & 1 & 0 & $\ldots$ \\
. & 0 & 0 & 0 & 1 & 0 & 0 & 0 & 1 & 0 & 0 & $\ldots$ \\
. & 1 & 0 & 1 & 0 & 1 & 0 & 1 & 0 & 1 & 0 & $\ldots$
\end{tabular}

3. The set of natural numbers $\mathbb{N}$,

$$
\mathbb{N}=\{1,2,3, \ldots\}
$$

4. The following arrangement matching each item in $\mathrm{L}$ with an element of

\begin{tabular}{|c|c|c|c|c|c|c|c|c|c|c|c|}
\hline $\mathrm{n}$ & & & & & d & & & & & & \\
\hline 1 & .0 & 1 & 0 & 1 & 0 & 1 & 0 & 1 & 0 & 1 & \\
\hline 2 & . 1 & 1 & 0 & 1 & 1 & 0 & 1 & 1 & 0 & 1 & \\
\hline 3 & .0 & 0 & 1 & 0 & 0 & 1 & 0 & 0 & 1 & 0 & \\
\hline 4 & 0 & 0 & 0 & 1 & 0 & 0 & 0 & 1 & 0 & 0 & \\
\hline 5 & . 1 & 1 & 1 & 0 & 1 & 1 & 1 & 0 & 1 & 1 & $\ldots$ \\
\hline 6 & 0 & 0 & 0 & 0 & 1 & 0 & 0 & 0 & 0 & 1 & \\
\hline 7 & . 1 & 1 & 1 & 1 & 0 & 1 & 1 & 1 & 1 & 0 & \\
\hline 8 & . 0 & 0 & 1 & 1 & 0 & 0 & 1 & 1 & 0 & 0 & \\
\hline 9 & . 1 & 1 & 0 & 0 & 1 & 1 & 0 & 0 & 1 & 1 & \\
\hline 10 & . 1 & 0 & 1 & 0 & 1 & 0 & 1 & 0 & 1 & 0 & $\ldots$ \\
\hline
\end{tabular}
$\mathbb{N}$ :

\section{Creating a number $Y$ not contained in $L$}

Next we use the diagonal method to construct a binary decimal number that is not in the list by flipping the first digit of the number in the first row, the second digit of the number in the second row, the third digit in the number in the third row and so on. The resulting table will appear as follows: 


\begin{tabular}{c|cccccccccccc}
$\mathrm{n}$ & & & & & & $\mathrm{d}$ & & & & & & \\
\hline 1 &. & 0 & 1 & 0 & 1 & 0 & 1 & 0 & 1 & 0 & 1 & $\ldots$ \\
2 &. & 1 & 1 & 0 & 1 & 1 & 0 & 1 & 1 & 0 & 1 & $\ldots$ \\
3 &. & 0 & 0 & 1 & 0 & 0 & 1 & 0 & 0 & 1 & 0 & $\ldots$ \\
4 &. & 0 & 0 & 0 & 1 & 0 & 0 & 0 & 1 & 0 & 0 & $\ldots$ \\
5 &. & 1 & 1 & 1 & 0 & 1 & 1 & 1 & 0 & 1 & 1 & $\ldots$ \\
6 &. & 0 & 0 & 0 & 0 & 1 & 0 & 0 & 0 & 0 & 1 & $\ldots$ \\
7 &. & 1 & 1 & 1 & 1 & 0 & 1 & 1 & 1 & 1 & 0 & $\ldots$ \\
8 &. & 0 & 0 & 1 & 1 & 0 & 0 & 1 & 1 & 0 & 0 & $\ldots$ \\
9 &. & 1 & 1 & 0 & 0 & 1 & 1 & 0 & 0 & 1 & 1 & $\ldots$ \\
10 &. & 1 & 0 & 1 & 0 & 1 & 0 & 1 & 0 & 1 & 0 & $\ldots$ \\
$\ldots$ & & & & & & $\ldots$ & & & & & & \\
\hline $\mathrm{Y}$ &. & $\mathbf{1}$ & $\mathbf{0}$ & $\mathbf{0}$ & $\mathbf{0}$ & $\mathbf{0}$ & $\mathbf{1}$ & $\mathbf{0}$ & $\mathbf{0}$ & $\mathbf{0}$ & $\mathbf{1}$ & $\ldots$ \\
& & & & & & & & & & & &
\end{tabular}

The number $Y$ will differ at each $n^{\text {th }}$ digit from the number in the $n^{\text {th }}$ row of the table. Therefore $Y$ cannot be in $L$ and cannot be matched with a natural number. Since $Y$ cannot be paired with a natural number not already in $L$, Cantor reasoned that the set of numbers in $(0,1)$ must be larger than the set of natural numbers.

\section{Changing the Initial Conditions}

We will construct a proof with slightly different initial conditions that will demonstrate that the infinite binary decimals in $(0,1)$ and natural numbers can be arranged in such a way as to match one natural number with one infinite binary decimal number in the interval without exception. Our new initial conditions are as follows:

1. The set $\mathbb{N}$ of natural numbers,

$$
\mathbb{N}=\{1,2,3, \ldots\}
$$

2. The set $\mathbb{N}_{e}$ of even natural numbers,

$$
\mathbb{N}_{e}=\{2,4,6, \ldots\}
$$

3. The set $\mathbb{N}_{o}$ of odd natural numbers,

$$
\mathbb{N}_{o}=\{1,3,5, \ldots\}
$$


4. From set theory we know that:

$$
\begin{gathered}
f: \mathbb{N} \rightarrow \mathbb{N}_{o}, \\
f: \mathbb{N} \rightarrow \mathbb{N}_{e}
\end{gathered}
$$

and

$$
\mathbb{N}_{o} \cup \mathbb{N}_{e}=\mathbb{N}
$$

therefore

$$
f: \mathbb{N} \rightarrow \mathbb{N}_{o} \cup \mathbb{N}_{e}
$$

5. The set $B$ of infinite binary decimals in the interval $(0,1)$,

$$
B=\{d: 0<d<1\}
$$

6. An infinite, incomplete list $L$, of the elements of $B$.

\begin{tabular}{llllllllllll}
\multicolumn{10}{c}{$\mathrm{d}$} \\
\hline & 0 & 1 & 0 & 1 & 0 & 1 & 0 & 1 & 0 & 1 & $\ldots$ \\
. & 1 & 1 & 0 & 1 & 1 & 0 & 1 & 1 & 0 & 1 & $\ldots$ \\
. & 0 & 0 & 1 & 0 & 0 & 1 & 0 & 0 & 1 & 0 & $\ldots$ \\
. & 0 & 0 & 0 & 1 & 0 & 0 & 0 & 1 & 0 & 0 & $\ldots$ \\
. & 1 & 0 & 1 & 0 & 1 & 0 & 1 & 0 & 1 & 0 & $\ldots$ \\
& & & & & & $\ldots$ & & & & &
\end{tabular}

We now begin as Cantor did, by constructing a number $Y$ not in $L$. Our list is constructed slightly differently than Cantor's in that we are matching each infinite binary decimal number in $L$ with an element of $\mathbb{N}_{o}$ as shown below: 


\begin{tabular}{|c|c|c|c|c|c|c|c|c|c|c|c|}
\hline $\mathrm{n}$ & & & & & d & & & & & & \\
\hline 1 & 0 & 1 & 0 & 1 & 0 & 1 & 0 & 1 & 0 & 1 & $\ldots$ \\
\hline 3 & 1 & 1 & 0 & 1 & 1 & 0 & 1 & 1 & 0 & 1 & $\ldots$ \\
\hline 5 & 0 & 0 & 1 & 0 & 0 & 1 & 0 & 0 & 1 & 0 & $\ldots$ \\
\hline 7 & 0 & 0 & 0 & 1 & 0 & 0 & 0 & 1 & 0 & 0 & $\ldots$ \\
\hline 9 & 1 & 1 & 1 & 0 & 1 & 1 & 1 & 0 & 1 & 1 & $\ldots$ \\
\hline 11 & 0 & 0 & 0 & 0 & 1 & 0 & 0 & 0 & 0 & 1 & $\ldots$ \\
\hline 13 & 1 & 1 & 1 & 1 & 0 & 1 & 1 & 1 & 1 & 0 & $\ldots$ \\
\hline 15 & 0 & 0 & 1 & 1 & 0 & 0 & 1 & 1 & 0 & 0 & $\ldots$ \\
\hline 17 & 1 & 1 & 0 & 0 & 1 & 1 & 0 & 0 & 1 & 1 & $\ldots$ \\
\hline 19 & 1 & 0 & 1 & 0 & 1 & 0 & 1 & 0 & 1 & 0 & $\ldots$ \\
\hline$\ldots$ & & & & & $\cdots$ & & & & & & \\
\hline $\mathrm{Y}$ & . 1 & 0 & 0 & & 0 & 1 & 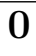 & 0 & 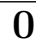 & 1 & \\
\hline
\end{tabular}

As before, $Y$ is not in $L$. The next step requires that we enter $Y$ into $L$. $L$ has no end so we put $Y$ in at the beginning of $L$ and match it with the first element of the set $\mathbb{N}_{e}$, which is 2 . See below.

\begin{tabular}{|c|c|c|c|c|c|c|c|c|c|c|c|}
\hline $\mathrm{n}$ & & & & & d & & & & & & \\
\hline 2 & 1 & 0 & 0 & 0 & 0 & 1 & 0 & 0 & 0 & 1 & \\
\hline 1 & 0 & 1 & 0 & 1 & 0 & 1 & 0 & 1 & 0 & 1 & $\ldots$ \\
\hline 3 & 1 & 1 & 0 & 1 & 1 & 0 & 1 & 1 & 0 & 1 & $\ldots$ \\
\hline 5 & 0 & 0 & 1 & 0 & 0 & 1 & 0 & 0 & 1 & 0 & $\ldots$ \\
\hline 7 & 0 & 0 & 0 & 1 & 0 & 0 & 0 & 1 & 0 & 0 & $\ldots$ \\
\hline 9 & 1 & 1 & 1 & 0 & 1 & 1 & 1 & 0 & 1 & 1 & $\ldots$ \\
\hline 11 & 0 & 0 & 0 & 0 & 1 & 0 & 0 & 0 & 0 & 1 & $\ldots$ \\
\hline 13 & 1 & 1 & 1 & 1 & 0 & 1 & 1 & 1 & 1 & 0 & $\ldots$ \\
\hline 15 & . 0 & 0 & 1 & 1 & 0 & 0 & 1 & 1 & 0 & 0 & $\ldots$ \\
\hline 17 & . 1 & 1 & 0 & 0 & 1 & 1 & 0 & 0 & 1 & 1 & $\ldots$ \\
\hline$\ldots$ & & & & & $\ldots$ & & & & & & \\
\hline $\mathrm{Y}$ & 0 & 0 & 1 & 1 & 1 & 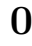 & 1 & 0 & 1 & 0 & \\
\hline
\end{tabular}

We repeat the procedure with the new number added to the list to create another number $Y$ also not in the list and match this number with the next element of $\mathbb{N}_{e}$ and add it to the list. Our list now looks like: 


\begin{tabular}{|c|c|c|c|c|c|c|c|c|c|c|c|}
\hline $\mathrm{n}$ & & & & & d & & & & & & \\
\hline 4 & 0 & 0 & 1 & 1 & 1 & 0 & 1 & 0 & 1 & 0 & $\ldots$ \\
\hline 2 & 1 & 0 & 0 & 0 & 0 & 1 & 0 & 0 & 0 & 1 & . \\
\hline 1 & 0 & 1 & 0 & 1 & 0 & 1 & 0 & 1 & 0 & 1 & $\ldots$ \\
\hline 3 & 1 & 1 & 0 & 1 & 1 & 0 & 1 & 1 & 0 & 1 & $\ldots$ \\
\hline 5 & 0 & 0 & 1 & 0 & 0 & 1 & 0 & 0 & 1 & 0 & $\ldots$ \\
\hline 7 & 0 & 0 & 0 & 1 & 0 & 0 & 0 & 1 & 0 & 0 & $\ldots$ \\
\hline 9 & 1 & 1 & 1 & 0 & 1 & 1 & 1 & 0 & 1 & 1 & . \\
\hline 11 & 0 & 0 & 0 & 0 & 1 & 0 & 0 & 0 & 0 & 1 & \\
\hline 13 & 1 & 1 & 1 & 1 & 0 & 1 & 1 & 1 & 1 & 0 & $\ldots$ \\
\hline 15 & 0 & 0 & 1 & 1 & 0 & 0 & 1 & 1 & 0 & 0 & . . \\
\hline$\ldots$ & & & & & $\ldots$ & & & & & & \\
\hline $\mathrm{Y}$ & 1 & 1 & 1 & & 1 & & 0 & & & & \\
\hline
\end{tabular}

This procedure can be repeated over and over indefinitely, expanding $L$ with infinite binary decimal numbers from $(0,1)$ not initially in $L$. After $i$ iterations our list looks like:

\begin{tabular}{c|cccccccccccc}
$\mathrm{n}$ & \multicolumn{110}{c}{$\mathrm{d}$} \\
\hline $\mathrm{n}$ & & & & \multicolumn{10}{c}{$\mathrm{Y}$} & & & & & & \\
$\ldots$ & & & & & & $\ldots$ & & & & & & \\
4 &. & 0 & 0 & 1 & 1 & 1 & 0 & 1 & 0 & 1 & 0 & $\ldots$ \\
2 &. & 1 & 0 & 0 & 0 & 0 & 1 & 0 & 0 & 0 & 1 & $\ldots$ \\
1 &. & 0 & 1 & 0 & 1 & 0 & 1 & 0 & 1 & 0 & 1 & $\ldots$ \\
3 &. & 1 & 1 & 0 & 1 & 1 & 0 & 1 & 1 & 0 & 1 & $\ldots$ \\
5 &. & 0 & 0 & 1 & 0 & 0 & 1 & 0 & 0 & 1 & 0 & $\ldots$ \\
7 &. & 0 & 0 & 0 & 1 & 0 & 0 & 0 & 1 & 0 & 0 & $\ldots$ \\
9 &. & 1 & 1 & 1 & 0 & 1 & 1 & 1 & 0 & 1 & 1 & $\ldots$ \\
11 &. & 0 & 0 & 0 & 0 & 1 & 0 & 0 & 0 & 0 & 1 & $\ldots$ \\
13 &. & 1 & 1 & 1 & 1 & 0 & 1 & 1 & 1 & 1 & 0 & $\ldots$ \\
15 &. & 0 & 0 & 1 & 1 & 0 & 0 & 1 & 1 & 0 & 0 & $\ldots$ \\
$\ldots$ & & & & & & $\ldots$ & & & & & & \\
\hline
\end{tabular}

We now have a situation where every element of $L$, at any moment in time, is matched with one and only one natural number. And going forward, no matter how many numbers $Y$ are added to the list, each will be matched with one and only one natural number. 


\section{Proof that $f: \mathbb{N} \rightarrow B$}

Let $B_{m}$ be defined as a subset of $B$ such that every element of $B_{m}$ is a member of $L$ before any number $Y$ has been created and inserted into $L$,

$$
B_{m} \subset B \text { and } B_{m}=\{d: d \in L\} .
$$

Let $B_{n}$ be defined as a subset of $B$ such that every element of $B_{n}$ is not a member of $L$ before any number $Y$ has been created and inserted into $L$,

$$
B_{n} \subset B \text { and } B_{n}=\{d: d \notin L\} .
$$

The union of $B_{m}$ and $B_{n}$ is the set $B$,

$$
B_{m} \cup B_{n}=B \text {. }
$$

For every $d \in B_{m}$ we have one and only one corresponding $n \in \mathbb{N}_{o}$ therefore

$$
f: \mathbb{N}_{o} \rightarrow B_{m} \text { exists. }
$$

For every $d \in B_{n}$ we have one and only one corresponding $n \in \mathbb{N}_{e}$ therefore

$$
f: \mathbb{N}_{e} \rightarrow B_{n} \text { exists. }
$$

It follows that

$$
\begin{gathered}
f:\left(\mathbb{N}_{o} \cup \mathbb{N}_{e}\right) \rightarrow\left(B_{m} \cup B_{n}\right) \text { exists and since } \\
\left(\mathbb{N}_{o} \cup \mathbb{N}_{e}\right)=\mathbb{N} \text { and }\left(B_{m} \cup B_{n}\right)=B \text { then } \\
f: \mathbb{N} \rightarrow B
\end{gathered}
$$

This ends the demonstration and completes the proof. 


\section{Appendix}

In the appendix we demonstrate that using the diagonal method recursively will, at the limit of the process, fully account for all the infinite binary decimals in $(0,1)$. The proof will cement the one-to-one correspondence between the natural numbers and the infinite binary decimals in $(0,1)$. Finally, we will propose a conjecture that the apparent different sizes of infinite sets are merely a matter of arrangement or the form of presentation and not actual differences.

\section{Initial Conditions}

1. The set $\mathbb{N}$ of natural numbers,

$$
\mathbb{N}=\{1,2,3, \ldots\}
$$

2. The set $\mathbb{N}_{e}$ of even natural numbers,

$$
\mathbb{N}_{e}=\{2,4,6, \ldots\}
$$

3. The set $\mathbb{N}_{o}$ of odd natural numbers,

$$
\mathbb{N}_{o}=\{1,3,5, \ldots\}
$$

4. From set theory we know that:

$$
f: \mathbb{N} \rightarrow \mathbb{N}_{o}, f: \mathbb{N} \rightarrow \mathbb{N}_{e} \text { and } \mathbb{N}_{o} \cup \mathbb{N}_{e}=\mathbb{N} \text { therefore } f: \mathbb{N} \rightarrow \mathbb{N}_{o} \cup \mathbb{N}_{e}
$$

5. The set $B$ of infinite binary decimals in the interval $(0,1)$,

$$
B=\{d: 0<d<1\}
$$

6. The infinite list $\mathrm{L}$ of elements of $B$,

\begin{tabular}{lllllllllll}
\hline . & $d_{1}$ & $d_{2}$ & $d_{3}$ & $d_{4}$ & $d_{5}$ & $d_{6}$ & $d_{7}$ & $d_{8}$ & $d_{9}$ & $\ldots$ \\
. & $d_{1}$ & $d_{2}$ & $d_{3}$ & $d_{4}$ & $d_{5}$ & $d_{6}$ & $d_{7}$ & $d_{8}$ & $d_{9}$ & $\ldots$ \\
. & $d_{1}$ & $d_{2}$ & $d_{3}$ & $d_{4}$ & $d_{5}$ & $d_{6}$ & $d_{7}$ & $d_{8}$ & $d_{9}$ & $\ldots$ \\
. & $d_{1}$ & $d_{2}$ & $d_{3}$ & $d_{4}$ & $d_{5}$ & $d_{6}$ & $d_{7}$ & $d_{8}$ & $d_{9}$ & $\ldots$ \\
. & $d_{1}$ & $d_{2}$ & $d_{3}$ & $d_{4}$ & $d_{5}$ & $d_{6}$ & $d_{7}$ & $d_{8}$ & $d_{9}$ & $\ldots$ \\
& & & & & & $\ldots$ & & & &
\end{tabular}

7. Let $B_{m}$ be defined as a subset of $B$ such that every element of $B_{m}$ is a member of $L$ before any number $Y$ has been created and inserted into $L$, 


$$
B_{m} \subset B \text { and } B_{m}=\{d: d \in L\} \text { therefore }\left(\forall d \in B_{m}\right) \in L
$$

8. Let $B_{n}$ be defined as a subset of $B$ such that every element of $B_{n}$ is not a member of $L$ before any number $Y$ has been created and inserted into $L$,

$$
B_{n} \subset B \text { and } B_{n}=\{d: d \notin L\} \text { therefore }\left(\forall d \in B_{n}\right) \notin L
$$

\section{Demonstration}

Since

$$
\left(\forall d \in B_{m}\right) \in L
$$

and each $\mathrm{d}$ in $L$ is matched with one and only one $n \in \mathbb{N}_{o}$ then

$$
f: \mathbb{N}_{o} \rightarrow B_{m}
$$

Since for each

$$
\left(d \in B_{n}\right) \notin L
$$

when constructed and entered into $L$ via the diagonal method, is matched with one and only one $n \in \mathbb{N}_{e}$ then

$$
f: \mathbb{N}_{e} \rightarrow B_{n}
$$

Let $Y_{i}$ represent a given $\left(d \in B_{n}\right)$ constructed via the diagonal method. $Y_{i}$ can then be viewed as a function of employing the diagonal method over the domain $L$. The function is recursive in nature since each $Y_{i}$ constructed becomes part of the domain for the next iteration.

$$
f\left(Y_{i}\right)=\operatorname{DM}\left(L+Y_{i-1}\right)
$$

We can now ask the question:

At the limit of the diagonal method process will $f\left(Y_{i}\right)=\operatorname{DM}\left(L+Y_{i-1}\right)$ capture all the elements of $B_{n}$ ? That is, what is the resolution of the following limit expression:

$$
\lim _{i \rightarrow \infty} f\left(Y_{i}\right)=\forall d \in B_{n}
$$




$$
\exists r \in B_{n} \neq f\left(Y_{i}\right)
$$

then we must conclude that

$$
\lim _{i \rightarrow \infty} f\left(Y_{i}\right)
$$

produces a list $L$ that is a subset of $B$ and that a one-to-one correspondence between $\mathbb{N}$ and $B$ cannot be constructed using the arrangement proposed in The Diagonal Paradox.

To prove that at the limit of the process $L$ is complete we must show that all $d \in B_{n}$ will be constructed using the diagonal method:

$$
D M\left(L+Y_{i-1}\right)=\forall d \in B_{n}
$$

and since

$$
f\left(Y_{i}\right)=D M\left(L+Y_{i-1}\right)
$$

we have

$$
f\left(Y_{i}\right)=\forall d \in B_{n}
$$

1. $f\left(Y_{i}\right)$ is a value in the range of the diagonal method applied recursively over the domain $L$ with $Y_{i-1}$ appended to the beginning of $L$

2. Therefore $Y_{i}$ is defined as $D M\left(L+Y_{i-1}\right)$, that is

$$
f\left(Y_{i}\right)=D M\left(L+Y_{i-1}\right)
$$

3. The complete range of $f(Y)$ is equal to the limit as $i \rightarrow \infty$ of the diagonal method applied to the entire domain $(L+\forall Y)$

$$
f(Y)=\lim _{i \rightarrow \infty} D M(L+\forall Y)
$$

4. Assume the existence of at least one $d \in B_{n}$ which is not in the range of $f\left(Y_{i}\right)$, that is

$$
\left(\exists d \in B_{n}\right) \neq f\left(Y_{i}\right)
$$

5. Let $\left(\exists d \in B_{n}\right) \neq f\left(Y_{i}\right)$ be denoted by $X$

6. If $X$ exists and is not created during the diagonal method procedure then the procedure must be finite with $X$ being the value of the limit at $\infty$. But then $X$ can simply be added to the beginning of $L$ and another $Y_{i}$ will be 
constructed kicking off the procedure once more. Since any $X$ will restart the procedure it stands to reason that the procedure is not finite and that all $d \in B_{n}$ will be created at the limit of the process and $L$ will be complete.

7. The completed list $L$ is then equal to the union of the sets $B_{m}$ and $B_{n}$ and we have $L=B_{m} \cup B_{n}$. But we have previously shown that $f: \mathbb{N}_{o} \rightarrow B_{m}$ is given and demonstrated that $f: \mathbb{N}_{e} \rightarrow B_{n}$. This appendix justifies the statement that the diagonal method recursively applied will, at the limit of the process, capture all $d \in B_{n}$ which supports the conclusion that

$$
f: N \rightarrow B
$$

as demonstrated in the main paper.

\section{Conclusion}

We have seen that by splitting the set of natural numbers into two subsets, the odd numbers and the even numbers, that we are able to construct an arrangement of symbols whereby a one-to-one correspondence between the natural numbers and the infinite binary decimal numbers in the open interval $(0,1)$. We made use of Cantor's diagonal method applied recursively in order to show that the list of infinite binary decimals can be considered complete at the limit of the diagonalization process. This result lends credence to the supposition that the success of one-to-one matching of infinite sets is more a matter of arrangement than anything to do with the "size" of one infinite set compared to another. 